\title{
A Study of Clincoetiological Profile of Patients with Pleural Effusion
}

\author{
Arya Shashikant, Gupta Archana \\ Department of Medicine, G.R. Medical College, Gwalior (M.P.) \\ Department of chest\&TB Jaya Arogya Hospital Gwalior (M.P.)
}

\begin{abstract}
Introduction: A pleural effusion is an abnormal collection of fluid in the pleural space resulting from excess fluid production or decreased absorption or both. It reflects an abnormal pathophysiological state resulting from disequilibria between pleural fluid formation and removal.

Objectives: The objective of this study was to determine the clinical profile, etiology in patients presenting with pleural effusion and correlation between etiology, clinical and radiological findings.

Material And Methods: It was a cross sectional study carried out at the GRMC JAH Department of Medicine, Gwalior, from to July 2013 to October 2014. Inclusion criteria were all 100 patients above 14 years of age with clinical and radiological evidence of pleural effusion. Patients with past history of trauma chest, thoracocentesis were excluded. The demographic variables were gender, age in years, sex and address. The research variables were breathlessness, fever, cough, chest pain, generalized edema, abdominal pain, haemoptysis and cause of pleural effusion. Investigations like CBC, RBS, RFT, serum albumin, CXR, and pleural fluid analysis, USG chest and abdomen, echocardiogram, UGI endoscopy, blood culture, FNAC and CT Scan. There were eight age groups between below 21 years to above 81 years. Nominal data was analyzed for frequency and (\%) and the numeric data was analyzes by chi square test p-value.

Results: In present study maximum cases (66\%) presented with exudative type of pleural effusion. Of these 43 cases $(65.15 \%)$ had tuberculosis, 5 cases (7.5\%) had malignancy 4 cases (6.06\%) had pneumonia.34\% cases presented with transudative type of pleural effusion. Of these 10 cases (29.41\%) had hepatic involvement, 8 cases (23.52\%) had CCF. Acute febrile illness 9(9\%), Nephrotic syndrome 4(16\%), renal failure 5(5\%), hypoalbuminemea $5(5 \%)$ pancreatitis $(1 \%)$ and ARDS (1\%) were other causes of pleural effusion in this study.

Keywords: Tuberculosis, Congestive cardiac failure, Adult Respiratory Distress Syndrome
\end{abstract}

\section{Introduction}

A pleural effusion is an abnormal collection of fluid in the pleural space resulting from excess fluid production or decreased absorption or both. It reflects an abnormal pathophysiological state resulting from disequilibria between pleural fluid formation and removal. It is the most common manifestation of pleural disease, with etiologies ranging from cardiopulmonary disorders to symptomatic inflammatory or malignant diseases requiring urgent evaluation and treatment. ${ }^{1}$

Pleural effusion is a common finding among patients presenting with cardiopulmonary symptoms. A systemic approach to the investigations is needed because of the extensive differential diagnosis. Pleural effusions can be transudative or exudative. ${ }^{2}$ In cases with transudative pleural effusion the diagnosis is usually made without much difficulties but exudative pleural effusion requires careful differential diagnosis that includes parapneumonic effusion, tuberculosis, and metastatic cancers which are found to be the cases in large number of patients. ${ }^{3,4}$ Pleural cavity is a potential space lined by mesothelium containing about $0.1-0.2 \mathrm{ml} / \mathrm{kg}$ of clear colourless fluid which is an ultrafiltrate derived from the capillaries of the parietal pleura.It is reabsorbed through the lymphatic and venules of visceral pleura. Pleural fluid has a specific gravity of $1.010-1.026, \mathrm{pH}$ of 6.8-7.6, protein of $1.5 \mathrm{~g} / \mathrm{dL}$ with an albumin $50-70 \%$ and globulin of $30-45 \%$.

\section{Aims And Objectives}

There are not many study done to know the clinic etiological profile of patients with pleural effusion in the Department Of Medicine G.R.M.C/JAH Tertiary Care Centre.

- To study the clinical profile of patients with pleural effusion.

- To study the aetiology of pleural effusion.

- To study the co-relation between aetiology and clinical and radiological finding in patients with pleural effusion.

\section{Materials And Methods}

In this study, a total of 100 patients admitted to GRMC/JAH Tertiary care Centre, with varied admitting diagnosis whose length of stay exceeded over 24hrs and satisfying the inclusion criteria and had 
pleural effusions at the time of diagnosis or those developing during the hospital stay were evaluated prospectively.INCLUSION CRITERIA: All 100 patients (> 14 years of age) with clinically or radiologically documented pleural effusion were included in present study.EXCLUSION CRITERIA 1. Patients with hydropneumothorax and Trauma chest will be excluded 2.Past history of thoracocentesis

The demographic data collected included age, sex, address. A detailed historical account was obtained with emphasis on chief complaint, history of presenting illness, significant past history including the drug history. A through physical examination was done. Investigations like complete heamogram, random blood sugar; renal function tests, serum albumin, chest-x-ray, and pleural fluid analysis were carried out in all the cases. Investigations like ultrasonogram of the chest and abdomen, echocardiogram, upper gastrointestinal tract endoscopy, blood culture, FNAC and CT Scan of chest and abdomen of the lung were carried out as optional investigations when relevant to supplement the diagnosis.

The pleural fluid was quantified as small, moderate and large based on the chest radiographic finding in the upright or semi-upright position: Small - If the fluid obliterates the lower lung zone. Moderate - lower and middle lung zones. Large- all three-lung zones. On decubitus radiography, the widest measurement of pleural fluid from the inner wall of the chest to the free fluid edge was used for quantification of the effusion. The effusion were said to be:Small - width is $<1.5 \mathrm{cms}$,Moderate - 1.5 to $4.5 \mathrm{cms}$, Large - $>4.5 \mathrm{cms}$.

The pleural fluid analysis was routinely done in all the cases and if the effusion was small it was carried out under ultrasonographic guidance. The pleural fluid analysis included the study of $\mathrm{pH}$, protein, cell count and type, gram staining, acid fast staining, and culture. we classified the effusions into a transudate or an exudates based on the Lights criteria, the effusion was said to be an exudates if they meet at least one of the following and transudates meet none of these criteria:1-Pleural fluid protein / serum protein $>0.5$ 2-Pleural fluid LDH / serum LDH > 0.6 3-Pleural fluid LDH more than two-thirds of normal upper limit for serum. When found to be an exudates further analysis to discern the cause of the effusion like pleural fluid cytology for malignant cells, pleural fluid adenosine deaminase, Polymerase chain reaction, amylase was carried out, as the case may need.In case of transudative effusions no further analysis of the pleural fluid was done.

\section{Results}

Out of 100 cases of pleural effusion $70(70 \%)$ were male and 30(30\%) female with male to female ratio 2.3 to 1.According to age, mean age was 38.10 years.Maximum no of cases was presented in 21-60 year of age because people are exposed to occupation hazards,smoking and infections, as the person is physically active. In the age group 21-60 years, $72 \%$ of cases falls in adult working group.According to past history 8 patients(36.40\%) had come with history of Pulmonary Tuberculosis which was highest as compare to other diseases.

According to symptoms, fever (53\%) and breathlessness $(45 \%)$ was most common symptoms followed by, cough, chest pain and abdominal pain. In our study maximum patients (50\%) presented with pallor which is a sign of chronic infection followed by pedal edema (23\%). According to X-ray findings small right sided pleural effusion are most common. we found right side cases more than left side. The P-Value is $<0.00001$. The result is significant at $\mathrm{p}<0.05$.

Table 1: Distribution of disease according to ADA level wise

\begin{tabular}{|l|c|c|c|c|}
\hline \multicolumn{1}{|c|}{ DIAGNOSIS } & $\mathbf{4 4 0}$ & $\mathbf{4 0 - 7 0}$ & $\mathbf{> 7 0}$ & Total \\
\hline TB & 8 & 12 & 25 & 45 \\
\hline MALIGNANCY & 0 & 4 & 2 & 06 \\
\hline HYPOPROTEINEMIA & 5 & 0 & 0 & 05 \\
\hline
\end{tabular}

Among cases of tubercular pleural effusion, there were 45 cases which showed greatly elevated ADA levels in pleural fluid ranging from $40 \mathrm{U} / \mathrm{Lit}$ to $>70 \mathrm{U} / \mathrm{Lit}$. In malignant effusion, all cases showed pleural fluid ADA value from $40 \mathrm{U}$ to $70 \mathrm{U} / \mathrm{Lit}$. All 5 cases of hypoproteinemia had value of pleural fluid ADA <40 U/Lit. As $p$ value is 0.00013 , statistically the result is significant. ADA values $>70 \mathrm{U} / \mathrm{L}$ are highly suggestive of tubercular etiology The values of pleural fluid ADA levels in tuberculosis were significantly raised when compared with malignant pleural effusion $(\mathrm{P}<0.0001)$, hypoproteinemia $(\mathrm{P}<0.0001)$.

Table 2: Distribution of study population according to type and etiology of pleural effusion

\begin{tabular}{|l|c|c|c|c|c|}
\hline \multicolumn{1}{|c|}{ Etiology of pleural effusion } & & Transudative & \% & Exudative & \% \\
\hline TB & 45 & 2 & $4.4 \%$ & 43 & $95.5 \%$ \\
\hline Hepatic & 13 & 10 & $76.9 \%$ & 3 & $23.1 \%$ \\
\hline CCF & 11 & 8 & $72.7 \%$ & 3 & $27.3 \%$ \\
\hline Acute febrile illness & 9 & 6 & $66.6 \%$ & 3 & $33.4 \%$ \\
\hline Malignancy & 6 & 1 & $16.7 \%$ & 5 & $83.3 \%$ \\
\hline Hypoalbuminemia & 5 & 3 & $60 \%$ & 2 & $40 \%$ \\
\hline Renal & 5 & 2 & $40 \%$ & $60 \%$ \\
\hline
\end{tabular}


A Study of Clincoetiological Profile of Patients with Pleural Effusion

\begin{tabular}{|l|c|c|c|c|c|}
\hline Pneumonia & 4 & 0 & 0 & 4 & $100 \%$ \\
\hline Pancreatitis & 1 & 1 & $100 \%$ & 0 & 0 \\
\hline ARDS & 1 & 0 & 0 & 1 & $100 \%$ \\
\hline Total & 100 & 34 & & 66 & \\
\hline
\end{tabular}

In present study maximum cases $(66 \%)$ presented with exudative type of pleural effusion. Of these 43 cases $(65.15 \%)$ had tuberculosis, 5 cases $(7.5 \%)$ had malignancy 4 cases $(6.06 \%)$ had pneumonia.

$34 \%$ cases presented with transudative type of pleural effusion. Of these 10 cases $(29.41 \%)$ had hepatic involvement, 8 cases $(23.52 \%)$ had CCF.

Type of fluid show a correlation with etiology of the effusion. Exudative pleural effusion is most common in tuberculosis43/45(95.5\%) while Transudative pleural effusion is most common in liver cirrhosis. The $p$ value is $<0.00001$ thus, Statistically result is significant at $\mathrm{p}<0.05$.

Table 3: Correlation of pleural effusion etiology with laterality

\begin{tabular}{|l|c|c|c|c|}
\hline \multirow{2}{*}{ Etiology of pleural effusion } & \multirow{2}{*}{ No of cases } & \multicolumn{3}{c|}{ Laterality } \\
\cline { 3 - 5 } & & BILATERAL & RIGHT & LEFT \\
\hline Tuberculosis & 45 & 11 & 21 & 13 \\
\hline Liver cirrhosis & 13 & 4 & 8 & 1 \\
\hline CCF & 11 & 9 & 4 & 3 \\
\hline Acute febrile illness & 9 & 2 & 2 & 2 \\
\hline Malignancy & 6 & 2 & 2 & 2 \\
\hline Hypoalbumenimia & 5 & 1 & 1 & 2 \\
\hline Renal & 5 & 2 & 3 & 1 \\
\hline Pneumonia & 4 & 0 & 0 & 0 \\
\hline Pancreatitis & 1 & 1 & 0 & 0 \\
\hline ARDS & 1 & 1 & & \\
\hline
\end{tabular}

$\mathrm{P}$ value laterality wise is 0.207 .the result is not significant at $\mathrm{p}<0.05$

Though it shows a co-relation but statistically not significant.

Table 4: Correlation of pleural effusion etiology with size

\begin{tabular}{|l|c|c|c|c|}
\hline \multirow{2}{*}{ Etiology of pleural effusion } & \multirow{2}{*}{ No of cases } & \multicolumn{3}{c|}{ Size } \\
\cline { 3 - 5 } & & Small & Moderate & 19 \\
\hline Tuberculosis & 45 & 26 & 5 & 0 \\
\hline Liver cirrhosis & 13 & 5 & 1 & 3 \\
\hline CCF & 11 & 9 & 4 & 0 \\
\hline Acute febrile illness & 6 & 5 & 3 & 0 \\
\hline Malignancy & 6 & 1 & - & 2 \\
\hline Hypoalbuminemia & 5 & 3 & 1 & - \\
\hline Renal & 5 & 4 & 0 & 0 \\
\hline Pneumonia & 4 & 4 & 0 & 0 \\
\hline Pancreatitis & 1 & 1 & 0 & 0 \\
\hline ARDS & 1 & 1 & & 0 \\
\hline
\end{tabular}

$\mathrm{P}$ value size wise is 0.009955.the result is significant at $\mathrm{p}<0.05$.

Correlation of size with disease is statistically highly significant and shows a strong predilection for the size of the effusion being directly proportional to etiology.

\section{Discussion}

In our study ,Maximum percentage of patients $70 \%$ were males and $30 \%$ were males and Male: Female ratio $2.3: 1$.

A.M. Abumossalam et al. in their study $72.3 \%$ were males and $27.7 \%$ were females and the male:female ration was 2.6:1.

In the present study, the mean age was $38.10 \pm 16.21$.The maximum number of cases $26 \%$ were in the age group 21-30 year followed by $20 \%$ cases were in the age group 31-40 years followed by $13 \%$ cases each for age group 41-50 and 51-60 age group .so in total maximum number of cases $72 \%$ were in the age group of 21-60 years

Mekonnen Desalew et al, 2002 The mean age was $37.0 \pm 15.69$.The maximum number of cases $55 \%$ were in the age group of 21-60 years. A.M.Abu Mossallam et al The mean age was 37.5 \pm 15.25 .The maximum number of cases $62 \%$ were in the age group of 21-60 years.

In present study, $36.4 \%$ of the cases had past history of pulmonary TB followed by $27.6 \%$ had history of diabetes mellitus followed by $13.6 \%$ each had history of CLD and CAD and remain in $1 \%$ each had history of RHD and HIV .Islam Farrukh,et al maxium percentage of the patients $43.75 \%$ had history of pulmonary TB followed by $28.12 \%$ had CAD followed by $12.5 \%$ had history of DM and $6.25 \%$ had history of CLD . 
In the present study maximum number of cases $53 \%$ had fever followed by breathlessness were present in $45 \%$ cases followed by cough in $31 \%$ cases and chest pain in $22 \%$ cases . In Mohd.Rehan.,et al Maximum number of the cases $82 \%$ had fever and breathlessness followed by $77 \%$ cases had cough and chest pain were present in $57 \%$ of the cases.

In present study Maximum $50 \%$ had signs of pallor followed by $23 \%$ had edema followed b y $14 \%$ had icterus then $13 \%, 7 \%$ and $1 \%$ had hepatolmegaly, spleenomegaly and cyanosis. Irappa Madabhavi., et al Maximum percentage $68 \%$ had signs of pedal edema followed by $65 \%$ had spleenomegaly followed by 45 $\%, 37 \%, 6 \%, 4 \%$ had pallor, icterus, hepatomegaly and cyanosis respectively .

In present study, maximum number of cases $66 \%$ had shown exudative type of pleural effusion followed by $34 \%$ had transudative type of pleural effusion. Results were similar to Mohd. Aurangzeb, et al study maximum number of cases $75 \%$ had shown exudative type of pleural effusion followed by $25 \%$ had transudative type of pleural effusion

In present study tubercular patients maximum 25 cases had ADA >70 12 cases had ADA level 40-70 and 8 cases had ADA <40 where as in hypoprotenimia patients all cases present had ADA level $<40$. RW Light et al 1999, ADA level more than $70 \mathrm{IU} / \mathrm{L}$ observed only in cases of tubercular pleural effusion so from the study we concluded that if ADA level of more than $70 \mathrm{IU} / \mathrm{L}$ is taken as cut off point it is exclusively seen in cases of tubercular pleural effusion. A proportion of ADA $\mathrm{p}<0.0001$ (statistically significant) is a good indicator of TB pleurisy, with an accuracy of $99 \%$, a sensitivity of $100 \%$, and a specificity of $98.6 \%$.

In present study maximum maximum cases $45 \%$ had right sided pleural effusion followed by $34 \%$ had bilateral pleural effusion and remaining $21 \%$ had left sided pleural effusion. M.E. Mohan, R George., et al.,2012 maximum number of patients $40 \%$ had right sided pleural effusion followed by $35 \%$ had bilateral pleural effusion and remaining $25 \%$ had left sided pleural effusion the results were comparable with our study.

In present study Maximum number of cases $45 \%$ had pulmonary tuberculosis followed by $13 \%$ of the cases were hepatic in etiology followed by $11 \%$ cases had CCF .In pulmonary TB patients out 45 cases 21 cases had right sided pleural effusion where as in CCF cases out of 11 cases 9 developed bilateral pleural effusion .

Luis Valdes et al., at a university hospital in Spain, they studied 642 Patients with pleural effusion revealed that the most frequent cause of the effusion was tuberculosis (25\%), followed by neoplasia $(22.9 \%)$, and congestive cardiac failure (17.9\%).86\% of these affected right side.

\section{Conclusion}

The aetiological distribution of pleural effusion depends on the geographic region, patient's age, and advances in diagnosis and treatment of the underlying cause.In our study we found :The commonest etiology of pleural effusion in patients is Tuberculosis followed by hepatic diseases and CCF in Department of Medicine GRMC/JAH Group of Hospital Gwalior. Most common cause of pleural effusion is Pulmonary Tuberculosis. Second most common cause of pleural effusion is Hepatic diseases. Right sided pleural effusion is most common in tuberculosis. In TB most effusions are small sized 58\%. Most common hepatic pleural effusion are right sided $61 \%$. CCF is third most common cause of pleural effusion $70 \%$ of effusion due to CCF are bilateral and $90 \%$ of all these effusions are small. $9 \%$ patients had pleural effusion due to acute febrile illness which resolved completely on treatment of underlying cause. Highest proportion of large size pleural effusion seen in malignancy 33\%. Most cases are in 21-60yrs age group. Most common presenting complaints are fever and breathlessness. Out of 100 cases, 70 male and 30 female patients have pleural effusion. Most common presentation in all 100 cases are right sided and small pleural effusion. Most of the effusions resolve with treatment of the underlying cause.Determining the aetiological and clinical pattern of pleural effusion helps in adoption of regionally optimized diagnostic and therapeutic attitudes

\section{References}

[1]. Diaz-Guzman E, Dweik RA. Diagnosis and management of pleural effusions: a practical approach. Compr Ther. Winter 2007;33(4):237-46

[2]. A.M.Abu Mossallam. Mohammad Rehan ."study of tb pleural effusion" 2013

[3]. Storey DD, Dines DE, Coles DT. Pleural effusion:a diagnostic dilemma. Journal of the American Medical Association. 1976;236:2183-6.

[4]. Keshmiri M, Hashemzadeh M. Use of cholesterol in differentiating of exudative and transudative pleural effusions. Medical journal of the Islamic Republic of Iran. 1997; 2(3):187-9.

[5]. $\quad$ Rowland, Belinda. "Pleural Effusion." Gale Encyclopedia of Cancer. The Gale Group Inc. 2002.Encyclopedia.com. 1 Nov. 2009.

[6]. Light RW, Macgegor MI, Luchsinger PC, et al. "Pleural Effusion: The diagnostic Separation of Transudates and Exudates."

[7]. John L Johnson, MD 2007:'Pleural effusion in cardiovascular disease pearls for correlating the evidence with the cause. PG Medicine/Vol 107/no 4 April 2007.

[8]. Sahn SA. Pleural effusions of extravascular origin. Clin Chest Med. Jun 2006;27(2):285-308.

[9]. Light RW. The undiagnosed pleural effusion. Clin Chest Med. Jun 2006;27(2):309-19.

[10]. Culotta R, Taylor D. Diseases of the pleura. In: Ali J, Summer WR, Levitzky MG, eds. Pulmonary Pathophysiology. $2^{\text {nd }}$ ed. New York: Lange Medical Books/McGraw-Hill; 2005:194-212.

[11]. Villena V, Lopez Encuentra, Echave-Sustaeta, Alvarez Martinez C, Martin Escribano Prospective study of 1,000 consecutive patients with pleural effusion. Etiology of the effusion and characteristics of the patients. Arch Bronconeumol. 2002 Jan;38(1):21-6. 
[12]. Etiology and Pleural Fluid Characteristics of Large and Massive Effusions Jose' Manuel Porcel; and Manuel Vives, (CHEST 2003; 124:978-983).

[13]. Is the pleural fluid transudate or exudate? A revisit of the diagnostic criteria J Joseph, P Badrinath, G S Basran, S A Sahn Thorax 2001;56:867-870

[14]. Role of Adenosine Deaminase Estimation in Differentiation of Tuberculous and Non-tuberculous Exudative Pleural Effusions Bharat Kumar Gupta Vinay Bharatb, Debapriya Bandyopadhyaya J Clin Med Res 2010;2(2):79-84.

[15]. WHO Global Tuberculosis Report 2013

[16]. Vinaya S Karkhanis, Jyotsna M Joshi Department of Respiratory Medicine T N Medical College and BYL Nair Hospital, Mumbai, India Pleural effusion: diagnosis, treatment, and management Open Access Emergency Medicine 2012:4 31-52.

[17]. Luis Valda, David Alvarez, et al. The etiology of pleural effusion in an area with high incidence of tuberculosis. CHEST/109/1/jan 1996/page 158-62.

[18]. John E. Heffner, and Jeffrey S. Klein. Recent Advances in the Diagnosis and Management of Malignant Pleural Effusions. Mayo Clinic Proceedings 2008 February;83(2):2235-250.

[19]. Anand Patel, Sushmita Choudhury. Tuberculous pleural effusion: clinico-radiological and biochemical features observed in an Indian region. Indian Journal of Medical Specialities 2011;2(2):144-146.

[20]. Prabhudesai PP, Mahashur AA, Mehta N, Ajay R. Exudative pleural effusions in patients over forty years of age--an analysis of seventy-six patients. J Post grad Med 1993;39:190. 